\title{
Linear-accelerator X-ray output: a multicentre chamber-based intercomparison study in Australia and New Zealand
}

\author{
M. A. Ebert ${ }^{1,2}$, S. J. Howlett ${ }^{3}$, K. Harrison ${ }^{4}$, D. Cornes $^{5}$, C. S. Hamilton ${ }^{6}$ and J. D. Denham ${ }^{4,7}$ \\ ${ }^{1}$ Department of Radiation Oncology, Sir Charles Gairdner Hospital, Nedlands, Australia \\ ${ }^{2}$ School of Physics, University of Western Australia, Perth, Australia \\ ${ }^{3}$ Australasian College of Physical Scientists and Engineers in Medicine, Mascot, Melbourne, Australia \\ ${ }^{4}$ Department of Radiation Oncology, Calvary Mater Newcastle, Newcastle, Australia \\ ${ }^{5}$ Trans-Tasman Radiation Oncology Group, Calvary Mater Newcastle, Newcastle, Australia \\ ${ }^{6}$ Heidelberg Repatriation Hospital, Victoria, Australia \\ ${ }^{7}$ School of Medicine and Population Health, University of Newcastle, Newcastle, Australia
}

\begin{abstract}
This paper describes the process and results of a survey of linear accelerator outputs as part of an Australasian Level III Dosimetry Intercomparison. This study involved the measurement of accelerator output under reference conditions ('Level I') with a small-volume ionisation chamber in water for 47 beams at 36 radiotherapy centres using the IAEA TRS 398 dose-to-water protocol. The mean ratio of measured to locally-determined accelerator output was $1.003 \pm 0.009$ (1 standard deviation) with a range from 0.981 to 1.024. No correlation could be found between output ratio and accelerator type or local output calibration protocol. The small-volume chamber used satisfied most requirements for the study though showed some variation in sensitivity via repeated cross-calibration with a chamber calibrated at a primary standards laboratory.
\end{abstract}

Key words TRS 398, radiotherapy, audit, dosimetry intercomparison

\section{Introduction}

Determining the absolute output of linear accelerators under reference conditions ['Level I' dosimetry ${ }^{1,2}$ ] is central to ascertaining the uniformity of radiotherapy dose delivery across multiple treatment centres. Such dosimetric 'intercomparison' studies are essential for:

- Assessing the uniformity of dose delivery across multiple centres - In assessing local standards, implementation of calibration protocols and consistency of dose delivery.

- Aiding the prevention of accidents and treatment misadministration - In conjunction with Level III $^{\mathrm{a}}$

Corresponding author: Martin Ebert, Department of Radiation Oncology, Sir Charles Gairdner Hospital, Hospital Ave, Nedlands WA 6009 Australia

Tel: +61 89346 4931, Fax: +61 893463402

Email: Martin.Ebert@health.wa.gov.au

Received: 25 July 2008; Accepted: 7 October 2008

Copyright (C) 2008 ACPSEM studies to assess the entire treatment process, indicates the contribution of machine output to dose delivery error ${ }^{3}$.

- Quality assurance of clinical trial data - Sensible extraction of data from clinical trials requires uniform dose delivery, or that the variation in dose delivery between centres be known ${ }^{4}$. Intercomparison of dose delivery across multiple sites provides an examination of the uniformity of dose delivery.

Several collaborative trials groups have undertaken and continue to undertake dosimetric Level I intercomparison studies to monitor uniformity of dose delivery. Many of these are based on the mailing of thermoluminescent dosimeters (TLDs) as part of dosimetric audits. Such audits have been provided for many years for assessment of dose delivery relating to clinical trials as well as for general quality-assessment purposes on a routine basis by several international groups:

\footnotetext{
${ }^{a}$ Here, 'Level III' Dosimetry is defined as dosimetric evaluation, using an anthropomorphic phantom, of the entire treatment chain from imaging to dose delivery and verification. This definition, used previously by Kron et al (2002), differs slightly from that defined by Izewska et al (2007), where a 'Level 4' study is equivalent to Level III
} 
- The International Atomic Energy Agency (IAEA) The IAEA has been conducting TLD-based audits since the 1960's ${ }^{2,5-9}$ b.

- The European Society for Therapeutic Radiology and Oncology (ESTRO)/European Organisation for Research and Treatment of Cancer (EORTC) European networks have provided extensive TLDbased audits to support trials, most recently via the Education, Science and QUality Assurance for Radiotherapy (ESQUIRE) project and the EQUAL facility ${ }^{4,10-12}$.

- The Radiological Physics Center (RPC) - The RPC undertakes TLD-based postal audits as well as chamber based output measurements via site visits, on behalf of multiple North American collaborative trials groups. ${ }^{c}$

- In the UK and Ireland, several national audits have been undertaken which include Level I studies ${ }^{13-15}$. Recently, a national trials support centre has been established to provide general QA and dosimetric support for trials ${ }^{16 \mathrm{~d}}$.

Level I studies have been limited since the discontinuation of IAEA audits ${ }^{17}$ in Australia in 2002. Ad hoc studies have been undertaken related to specific treatment sites, the introduction of new techniques or specific clinical trials ${ }^{1,18-20}$. The Australian Radiation Protection and Nuclear Safety Agency (ARPANSA), the local Primary Standard Dosimetry Laboratory (PSDL), undertook a posted TLD-based audit during 2002-2003, covering 36 accelerator beams at 17 institutions ${ }^{21}$. This indicated Level I dose delivery to be within $\pm 3.0 \%$ for those beams. Although no routine formal intercomparison process is established in Australia , individual centres can and have accessed the Level I dosimetry services provided on request from ARPANSA, the IAEA and other international groups, such as the RPC and the Children's Oncology Group ${ }^{\mathrm{f}}$.

The New Zealand National Radiation Laboratory (NRL) undertakes routine audits of linear accelerator outputs in New Zealand centres via chamber-based measurements. 62 measurements during 1999-2008 indicate a mean difference in output between the measurement and local output estimate of $-0.1 \% \pm 0.8 \%$ ( $1 \mathrm{SD}$ ) with a range of $-1.6 \%$ to $2.0 \% .^{\mathrm{g}}$

Australian and New Zealand centres calibrate accelerator output according to the IAEA protocols. Until the development of the absorbed dose-to-water protocol TRS $398^{22}$, calibration was principally according to airkerma $^{23}$ with each centre having chambers calibrated at a nearby standards laboratory - either ARPANSA or the New Zealand NRL. Since 1996, ARPANSA has provided absorbed dose-to-water calibrations against a primary standard. NRL maintains an absorbed dose-to-water standard traceable to ARPANSA. NRL have subsequently been able to provide absorbed dose-to-water calibrations since 2000.

We have had the opportunity to undertake a Level I dosimetry study at 36 radiotherapy centres across Australia and New Zealand. This study formed part of a larger investigation into the logistics and feasibility of establishing a dosimetric intercomparison service for Australia, and included a Level III dosimetric intercomparison with a pelvic phantom ${ }^{24,25}$. This comprehensive Level I-III audit of dosimetry in Australia followed up on recommendations from Kron et $\mathrm{al}^{1}$. The study was extended to include New Zealand centres due to the nature of multi-centre clinical trials undertaken in the region.

The dosimetric study enabled a single ionisation chamber/electrometer combination to be used to measure the absolute output of 47 megavoltage X-ray beams using the TRS 398 absorbed dose-to-water protocol ${ }^{22}$.

\section{Methods and materials}

\section{Study description}

Measurements were undertaken at the 36 participating radiotherapy centres between October 2004 and March 2008. A detailed summary of the process of undertaking the combined Level I/III study has been published elsewhere ${ }^{24}$. The reference centre was the Newcastle Mater Misericordiae Hospital ${ }^{\mathrm{h}}$ (NMMH) in New South Wales (NSW), approximately $150 \mathrm{~km}$ north of Sydney. All calibrations of measurement equipment were undertaken at the NMMH and physicists from this site, professionally accredited with the Australasian College of Physical Scientists and Engineers in Medicine ( $\mathrm{SH}$ or $\mathrm{ME}$ ), undertook all Level I measurements. Travel to centres in NSW was by motor vehicle, and to all other centres was by combination of passenger aircraft and motor vehicle. Each participating centre (other than the $\mathrm{NMMH}$ ) was visited only once.

Action levels were established prior to the study in order to provide objective criteria for immediate assessment of output measurement. The action levels and their criteria are defined in Table 1 . These are designed for consistency with results of previous studies, ${ }^{1,20}$, and to reflect the combined uncertainty in measurement of output with the equipment used in the study and that used locally at each centre. Centre results were not provided to any third party.

\section{Equipment}

As this study required transporting equipment by air and ground transport over large distances, equipment was required that was light and robust, yet which could be used to undertake output measurements consistent with the

\footnotetext{
${ }^{b}$ http://www-naweb.iaea.org/nahu/dmrp/tld.asp

${ }^{c}$ http://rpc.mdanderson.org/RPC/home.htm

${ }^{d}$ http://www.rttrialsqa.org.uk/

${ }^{e}$ Many centres collaborate to access independent measurement of accelerator output, especially following the installation of new accelerators. Additionally, some regions (e.g., Queensland and Victoria) have semi-formal processes in place to assess accelerator calibration

$f_{\text {http://www.childrensoncologygroup.org/ }}$

${ }^{g}$ Personal communication, Johnny Laban, New Zealand National Radiation Laboratory, March 2008

${ }^{h}$ Now the 'Calvary Mater Newcastle'
} 
Table 1. Action limits for ratio of measured accelerator output to locally-supplied output ( $O P_{\text {measured }} / O P_{\text {local }}$ ) established prior to study measurements on the basis of expected uncertainty in measurements, output tolerance standards and the results of previous studies.

$$
O P_{\text {measured }} / O P_{\text {local }} \quad \text { Action }
$$

Within range [0.98, 1.02] Within tolerance

Within range $[0.97,0.98) \quad$ Within tolerance but

or $(1.02,1.03] \quad$ measurement repeated once

Outside range [0.97, 1.03] $\begin{aligned} & \text { Outside tolerance. } \\ & \text { Investigate until resolved }\end{aligned}$

Table 2. Equipment used during the study.

\begin{tabular}{ll}
\hline Equipment & Description \\
\hline Electrometer & $\begin{array}{l}\text { Dose1 (Scanditronix-Wellhöfer, } \\
\text { Uppsala) s/n 038735 }\end{array}$ \\
Chamber & $\begin{array}{l}\text { CC13 (Scanditronix-Wellhöfer, } \\
\text { Uppsala) s/n 5441 }\end{array}$ \\
Aneroid barometer & $\begin{array}{l}\text { L991237 Precision Barometer } \\
\text { Digital }\end{array}$ Weatherstation with waterproof \\
thermometer & $\begin{array}{l}\text { transducer } \\
\text { In-house constructed, 30 cm x 30 } \\
\text { cm x 30 cm with fitting to hold } \\
\text { CC13 chamber at up to 20 cm depth } \\
\text { and levelling feet }\end{array}$ \\
\hline
\end{tabular}

requirements of the TRS 398 protocol $^{22, \mathrm{i}}$. The equipment selected is listed in Table 2.

A wooden box was constructed to transport the water tank, electrometer and accessories. This box was checked in as personal luggage on flights. The chamber itself was carried in its vendor-supplied box in the carry-on luggage of study members.

\section{Equipment calibration}

The CC13 chamber was cross-calibrated with a reference chamber at approximately three to six month intervals throughout the entire study, following the method detailed in TRS $398^{22}$ using a fixed SSD arrangement. Three megavoltage X-ray beams [nominal energies $4 \mathrm{MV}$ (Varian 600C s/n 434; $\mathrm{TPR}_{20,10}$ 0.624), 6 and $18 \mathrm{MV}$ (Varian 21EX s/n 2154; $\mathrm{TPR}_{20,10} 0.663$ and 0.780 respectively)] were available at the NMMH. The reference chamber was a Farmer-type 2571 thimble chamber and NE 2570 electrometer with an absorbed dose-to-water calibration coefficient from a ${ }^{60}$ Co beam $\left(N_{D, w}\right)$ traceable to the Australian primary standards laboratory at ARPANSA. Recombination $\left(k_{s}\right)$ and polarity effect $\left(k_{\text {pol }}\right)$ factors were measured for both the CC13 and NE 2571 chambers at each cross-calibration and removed from the calibration coefficient. Additionally, $k_{Q}$ values for the 2571 and CC13 chambers were obtained from TRS 398 in order to determine $N_{D, w}$ for the CC13 chamber. The constancy of the NE 2571 chamber was verified at least 6-monthly via a ${ }^{90} \mathrm{Sr}$ check. The CC13 chamber was cross-calibrated at 3 to 6 month intervals (depending on the frequency of site visits), with a delay of 16 months resulting from staff relocation and a lull in site visits towards the end of the study.

\section{Level I measurements}

Measurements were performed according to TRS 398, with the resulting output estimate, $O P_{\text {measured }}$, being for the local configuration and specification of dose in Gy per nominal Gy. A range of local output reference configurations were met, including combinations of fixed source-to-surface (SSD) and isocentric (SAD) output definitions, and output normalisation depth, $\boldsymbol{Z}_{\text {norm }}$, (where 1 accelerator monitor unit corresponds nominally to 1 cGy) from $Z_{\max }$ (depth of dose maximum) to $100 \mathrm{~mm}$. For all centres, the reference field size was $10 \mathrm{~cm} \mathrm{x} 10 \mathrm{~cm}$ (dimensions defined at isocentre). For SSD configurations, beam quality was determined from a measurement of $\mathrm{PDD}_{20,10}$ converted to $\mathrm{TPR}_{20,10}$ using the empirical relationship ${ }^{26}$

$T P R_{20,10}=1.2661 \cdot P D D_{20,10}-0.0595$

The depth of the chamber for reference dose measurement, $z_{\text {ref }}$, was $100 \mathrm{~mm}$, unless the local calibration was performed with an SAD arrangement with $Z_{\text {norm }}=50$ $\mathrm{mm}$, in which case a $z_{r e f}$ of $50 \mathrm{~mm}$ was used. The only factor not directly measured during the Level I measurement was the relative depth-related dose factor (i.e., $P D D_{z_{\text {ref }}, z_{\text {norm }}}$ or $T P R_{z_{\text {ref }}, z_{\text {norm }}}$ ), required for relating dose at $Z_{\text {ref }}$ to that at $Z_{\text {norm }}$.

Due to a slight variation in $N_{D, w}$ (absorbed-dose-towater calibration coefficient in ${ }^{60} \mathrm{Co}$ ), discovered for the CC13 chamber over time and with beam quality used for cross-calibration, the value used at the time of the Level I measurement at each centre was an interpolation (in beam quality) of the values obtained during the previous most recent cross-calibration of the chamber at the NMMH. $k_{s}$ and $k_{\text {pol }}$ were measured at each centre for the CC13 chamber and use to correct the chamber measurement. A $k_{Q}$ value for the chamber was determined from the quality measurement and the tables of calculated $k_{Q}$ values in TRS 398.

Extensive demographic data were collected to complement the Level I measurement, including a local

${ }^{i}$ Note that the CC13 chamber is covered in TRS 398 after version 10 of the document

${ }^{j}$ In this paper, measurements referred to as 'local' refer to measurements or data supplied by staff at each visited centre. Otherwise, the measurement referred to is that made by the study team during each centre's visit 


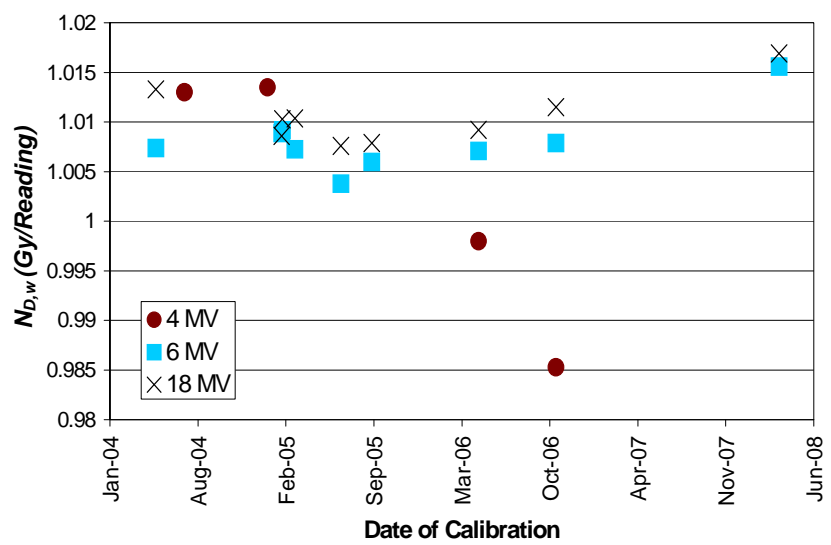

(a)

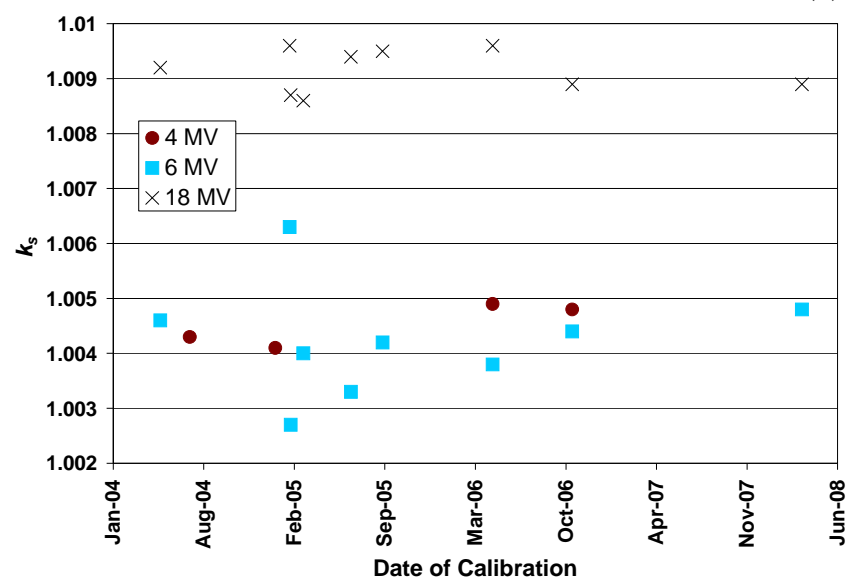

(b)

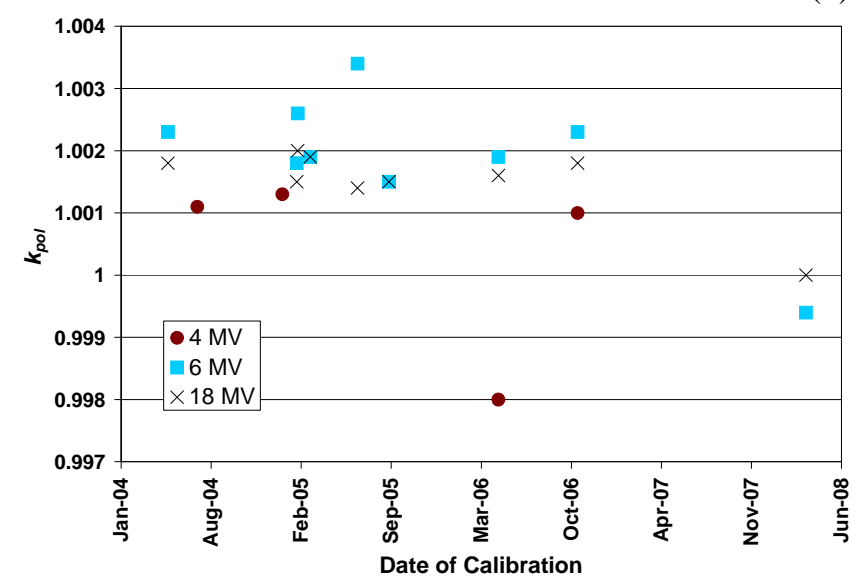

(c)

Figure 1. Variation in CC13 calibration characteristics obtained during cross-calibrations at the NMMH throughout the study. a) Dose to water calibration coefficient, $N_{D, w}$. b) Recombination correction factor, $\left.k_{s} . c\right)$ Polarity correction factor $k_{\text {pol }}$.

estimate of accelerator output, $O P_{\text {local }}$, which was provided by a local physicist. Pressure and temperature correction factors $\left(k_{T P}\right)$ were obtained from pressure and temperature measurements undertaken with the study equipment, though pressure measurements were also made using a local barometer for later comparison.

\section{Uncertainty analysis}

Uncertainties in Level I measurements will include contributions from quantities obtained at the time of the measurement, as well as from the cross-calibration of the CC13 chamber at the NMMH. TRS $398^{22}$ provides an estimate of a standard uncertainty in absolute dose determination of approximately $1.2 \%$ (correction factors determined by the PSDL at ARPANSA). Inclusion of the uncertainty in cross-calibration of the CC13 chamber will increase this by approximately $0.2 \%{ }^{22}$. If an additional (uncorrelated) $0.5 \%$ uncertainty is allowed from the local measurement of $P D D_{z_{\text {ref }}, z_{\text {norm }}}$ or $T P R_{z_{\text {ref }}, z_{\text {norm }}}$, then a standard uncertainty of approximately $1.5 \%$ can be expected in the Level I measurement. This should be considered in relation to the following results where error bars have not been included. This uncertainty is consistent with the recent analysis of Castro et $\mathrm{al}^{27}$, where an uncertainty of approximately $1.3 \%$ (in the absence of a cross-calibration) is estimated for the dose determination.

\section{Results}

\section{Characteristics of measurement equipment}

Figure 1 indicates the variation in factors for the CC13 chamber throughout the course of the study, obtained during the multiple cross-calibrations with the reference NE 2571 Farmer-type chamber. The equipment remained in good working order throughout the study. Leakage and electrometer voltage tests remaining consistent. This was despite damage to the transport box indicating some rough treatment during baggage handling.

\section{Demographics and measurement results}

\section{Accelerator types}

Table 3 provides a breakdown of linear accelerator equipment according to manufacturer and model. Measurements were performed on a single accelerator at all centres except one. No correlation was found between accelerator type and dosimetric result.

Table 3. Distribution (by manufacturer and model) of the accelerators on which output measurements were made.

\begin{tabular}{lll}
\hline Manufacturer & Model & Number of centres \\
\hline Varian (26) & $6 \mathrm{EX}$ & 2 \\
& 21EX & 8 \\
& 1800C & 1 \\
& 2100C & 10 \\
& 23EX & 2 \\
& 21IX & 3 \\
Philips (1) & SL25 & 1 \\
Elekta (5) & SL75 & 2 \\
& SL15 & 1 \\
& Precise & 2 \\
Siemens (5) & Primus & 2 \\
& KD2 & 1 \\
& Oncor & 2 \\
\hline
\end{tabular}




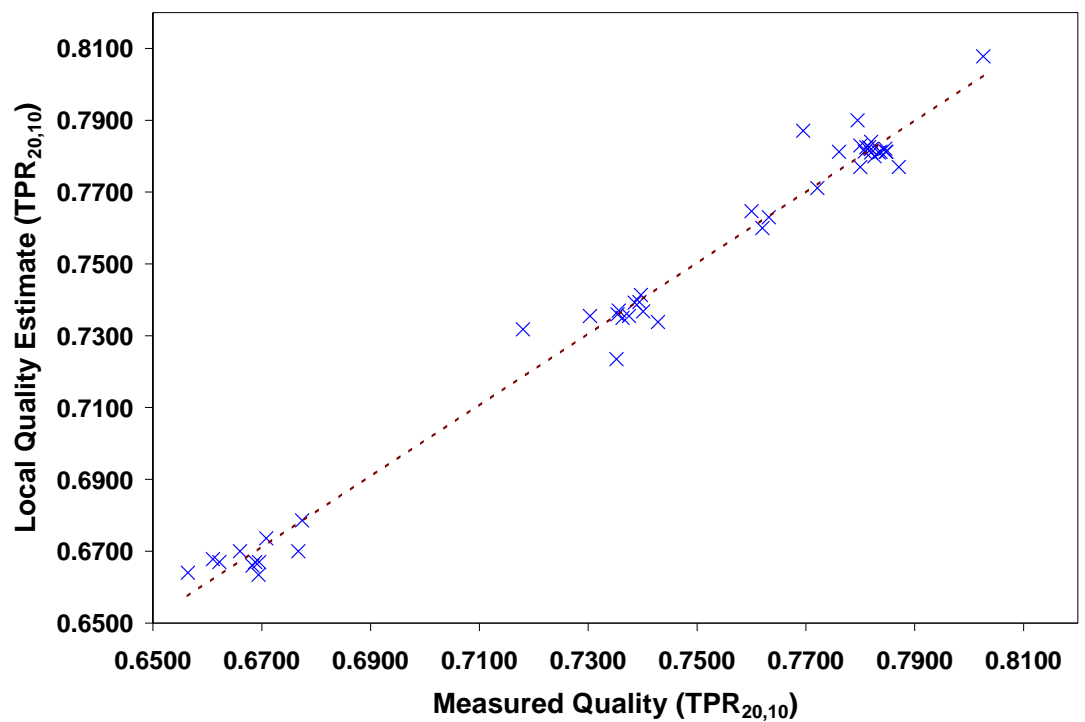

Figure 2. Distribution of local recorded beam quality $\left(T P R_{20,10}\right)$ against measured beam quality. Regression line $r^{2}=0.986$.

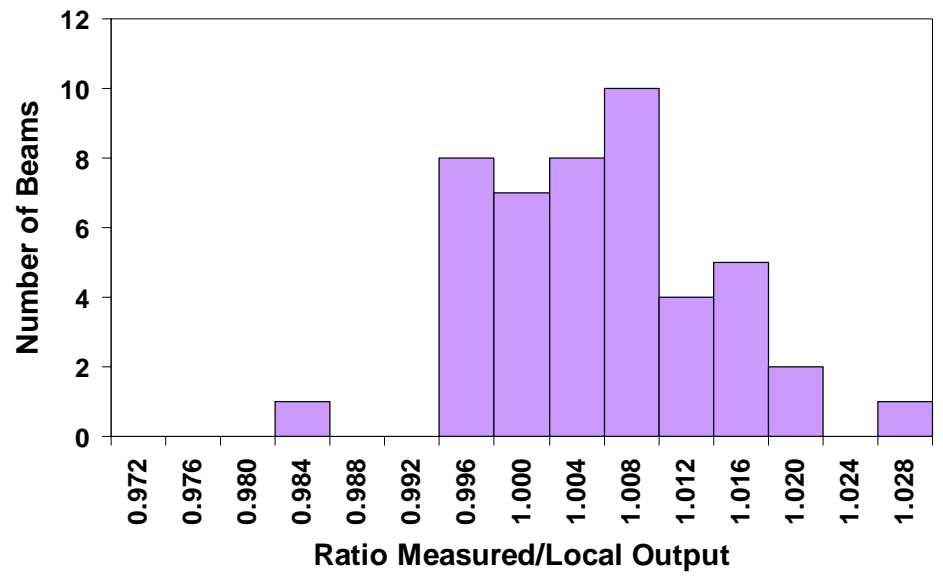

Figure 3. Histogram of ratios of measured accelerator output to local output estimation $\left(\mathrm{OP}_{\text {measured }} / \mathrm{OP}_{\text {local }}\right)$. Value on category axis is top of interval.

\section{Beam qualities}

Figure 2 shows the variation in beam quality specification according to local records relative to that determined during the Level I measurement. The mean percentage difference (relative to the local value) was -0.1 $\pm 0.7 \%$ (range $-2.2 \%$ to $+1.6 \%$ ).

\section{Accelerator output}

Results of accelerator output measurements are presented in terms of dose, in Gy per nominal Gy, at $z_{\text {norm }}$ according to local output configuration (ie., SSD or SAD). All accelerators considered had a nominal $100 \mathrm{~cm}$ source to axis (isocentre) distance. In all cases, $z_{\text {norm }}$ was defined as the point at which one accelerator monitor unit delivered nominally $1 \mathrm{cGy}$ for the local output configuration. Results for output, $O P$, are presented as

$O P=$ (Output Value) $\pm(1 \mathrm{SD})$ [minimum, maximum] Gy/nominal Gy
The mean local value for output, $\overline{O P_{l o c a l}}$, was:

$\overline{O P_{\text {local }}}=1.001 \pm 0.006[0.987,1.017] \mathrm{Gy} /$ nominal Gy.

In several cases, centres had calibrated their accelerator prior to the Level I visit and the local output value provided to the study team in such a case was $O P_{\text {local }}=1.000$ Gy/nominal Gy. The mean measured output for all Level I measurements performed by the study team, $\overline{O P_{\text {measured }}}$ was:

$\overline{O P_{\text {measured }}}=1.005 \pm 0.009[0.981,1.020] \mathrm{Gy} /$ nominal Gy.

Figure 3 provides a histogram comparison of the ratio of the measured accelerator output to the local output estimate. The mean ratio was:

$\overline{O P_{\text {measured }} / \text { OP }_{\text {local }}}=1.003 \pm 0.009[0.981,1.024]$. 


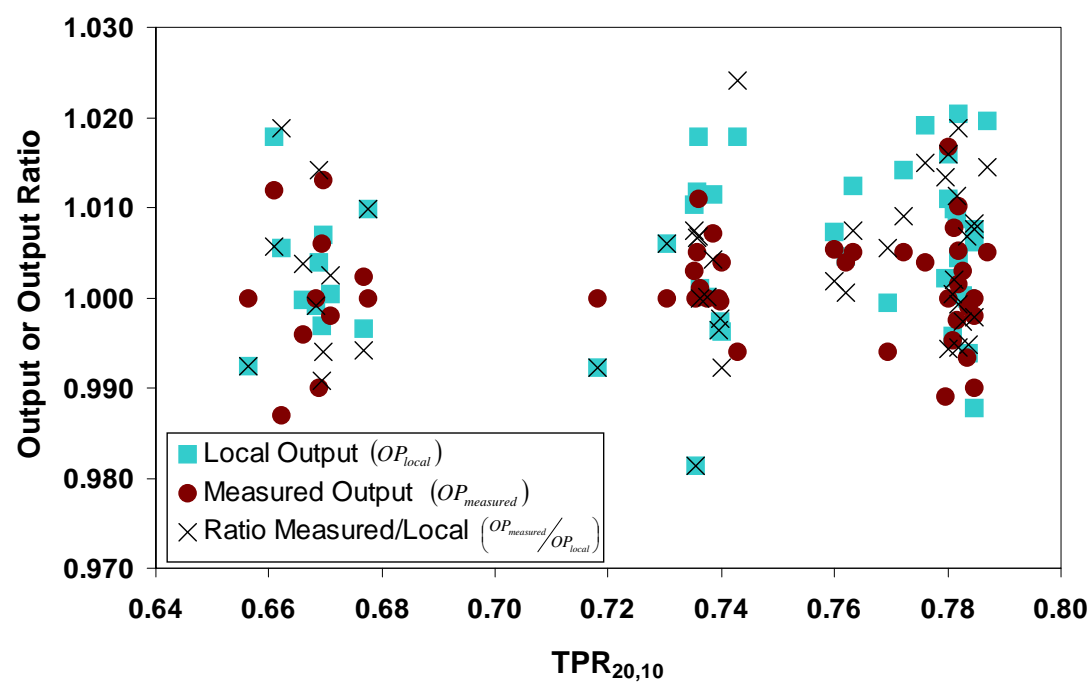

Figure 4. Accelerator outputs $\left(O P_{\text {local }}, O P_{\text {measured }}\right.$ and $\left.O P_{\text {measured }} / O P_{\text {local }}\right)$ versus beam quality.

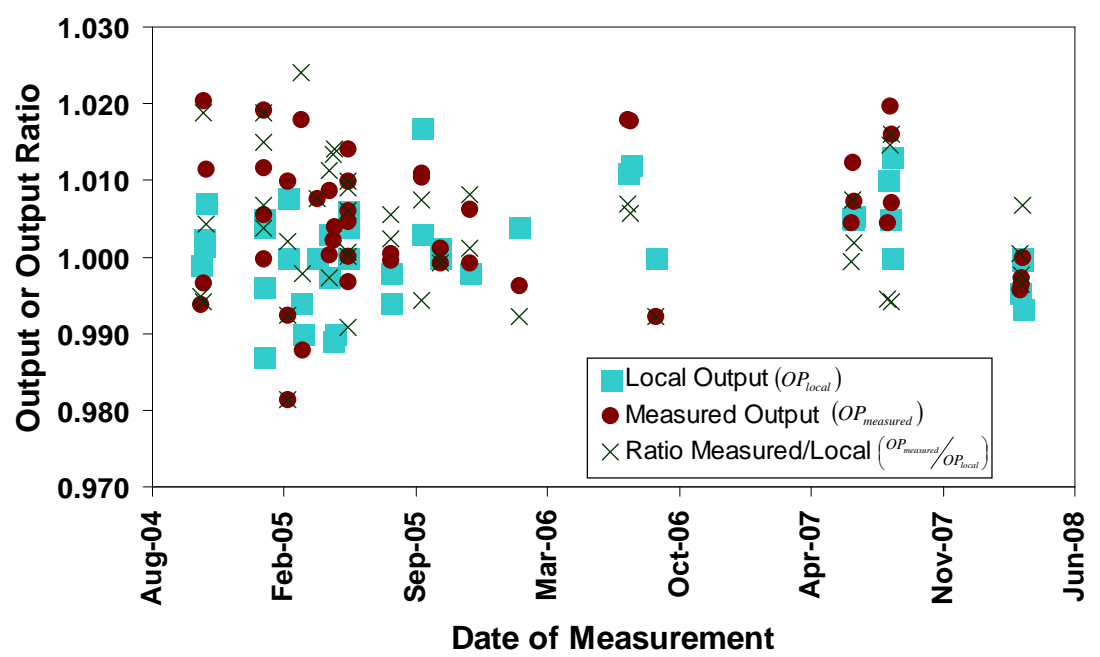

Figure 5. Accelerator outputs $\left(O P_{\text {local }}, O P_{\text {measured }}\right.$ and $\left.O P_{\text {measured }} / O P_{\text {local }}\right)$ ordered by measurement date.

Table 4. Summary of definitions of accelerator output employed by visited centres. All centres defined accelerator output for a 10 $\mathrm{cm} \times 10 \mathrm{~cm}$ field (size defined at isocentre). All accelerators had an $S A D$ of $100 \mathrm{~cm} . z_{\text {norm }}$ is the depth on central axis where the dose normalisation is specified (ie., where a dose of $1 c G y$ is nominally expected per monitor unit).

\begin{tabular}{ll}
\hline Definition & Number of centres \\
\hline SSD, $z_{\text {norm }}$ at $z_{\max }$ & 25 \\
SAD, $z_{\text {norm }}$ at $z_{\max }$ & 6 \\
SAD, $z_{\text {norm }}$ at 50 or $100 \mathrm{~mm}$ & 5 \\
\hline
\end{tabular}

Figuer 4 shows the distribution of measured outputs according to beam quality. Figure 5 provides the same data ordered by measurement date.

\section{Local output specification}

Table 4 shows the number of centres using each output specification and configuration encountered during the study. No correlation was found between local output specification and Level I measurement results.

\section{Local calibration protocol}

The protocol used at each centre was always either an Australasian adaptation of the IAEA TRS 277 air-kerma based protocol ${ }^{23}$, or the IAEA TRS 398 absorbed dose-towater protocol ${ }^{22}$. Australian and New Zealand centres have been gradually changing from TRS 277 to TRS 398 since 2000. The progression of centres to adoption of the TRS 398 protocol is indicated in Figure 6.

The variation in Level I results with local protocol is shown in Table 5. There was no significant difference 


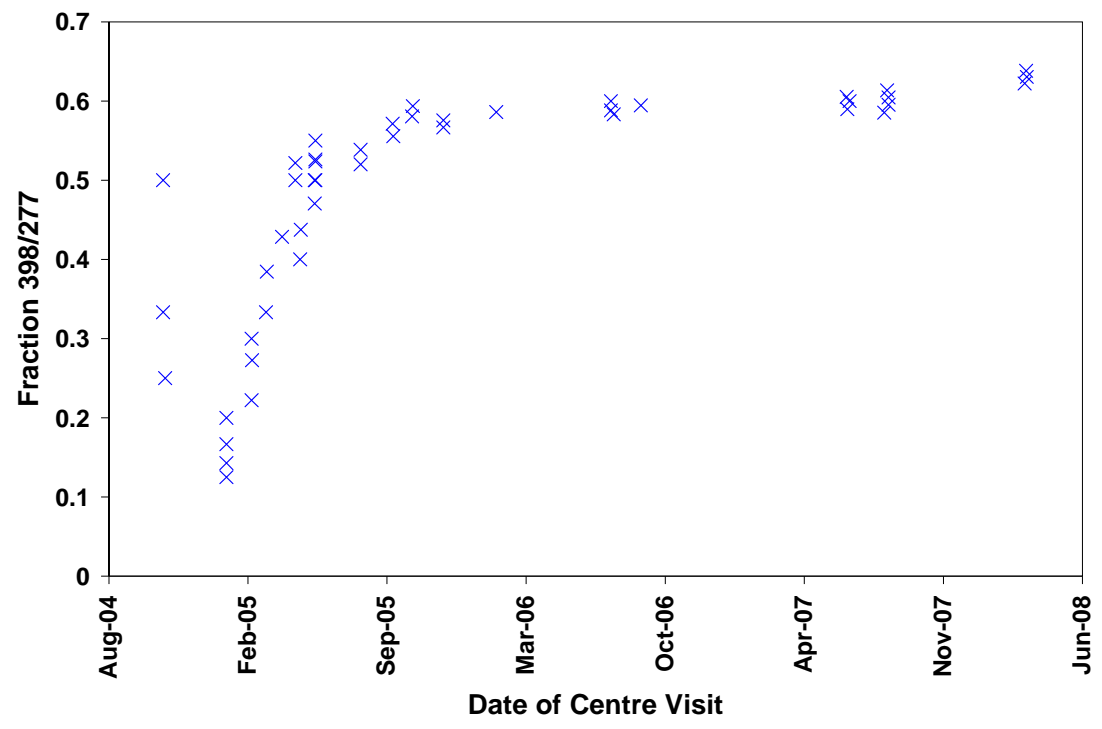

Figure 6. Time variation in the cumulative fraction of centres visited that were using the TRS 398 absorbed-doe-to-water based absolute dose protocol relative to the TRS 277 airkerma based protocol.

Table 5. Distribution of dose measurement protocol use across centres and distribution of Level I (accelerator output) results grouped by local protocol use.

\begin{tabular}{lll}
\hline & Centre uses TRS277 & Centre uses TRS398 \\
\cline { 2 - 3 } Number of centres & 13 & 23 \\
$\overline{O P_{\text {local }}} j \pm S D$ & $1.002 \pm 0.005$ & $1.001 \pm 0.007$ \\
$\overline{O P_{\text {measured }}} \pm S D$ & $1.006 \pm 0.009$ & $1.004 \pm 0.009$ \\
$\overline{O P_{\text {measured }} / \text { OP }_{\text {local }} \pm S D}$ & $1.004 \pm 0.009$ & $1.003 \pm 0.009$ \\
\hline
\end{tabular}

between local output estimates, those measurements made during the Level I visit or the difference between these two, nor between centres using either IAEA protocol.

\section{Discussion}

The choice of a small-volume chamber for this study was related to the design of the anthropomorphic phantom for the Level III study, which represented an adult male pelvis $^{25}$. This phantom was sectioned in horizontal slices and incorporated a recess for a CC13 chamber, centred on the centre-of-volume of the prostate where a dose measurement was required over a volume which was small relative to the size of the prostate and the expected dose modulation across it. The CC13 chamber was selected for the phantom as it is small, robust and transportable. A larger and less robust chamber would have been easily damaged during the study. The CC13 had the additional advantages of being waterproof and referenced in TRS 398 as a suitable chamber for absolute dosimetry. It could be accurately compared to an ARPANSA-calibrated chamber at the NMMH and then used in all other centres. This eliminated the need to transport two chambers and the additional requirement to reference the $\mathrm{CC} 13$ to the reference output at each centre. Figure 1 indicates some variation in $N_{D, w}$ with time which led to added uncertainties of the order of $0.3 \%$. The reason for the very low value of $N_{D, w}$ for the $4 \mathrm{MV}$ beam (see Figure 1a) is not apparent, though setup error cannot be excluded as a possible cause. Note that all beams measured in this study had nominal energies of $6 \mathrm{MV}$ or greater. Ideally the small volume chamber would be calibrated directly at a standards laboratory followed by periodic radioactive standards checks, and this is recommended for any future studies. Additionally, the variation in $N_{D, w}$ necessitated the application of the interpolation mentioned previously. Increasing Level I accuracy by using a larger volume chamber (such as a waterproof Farmer-type chamber which would be suitably robust for frequent transport), would be possible, though would limit the spatial resolution achievable in the phantom which may be particularly important if modulated (ie., IMRT) beams were to be used. 


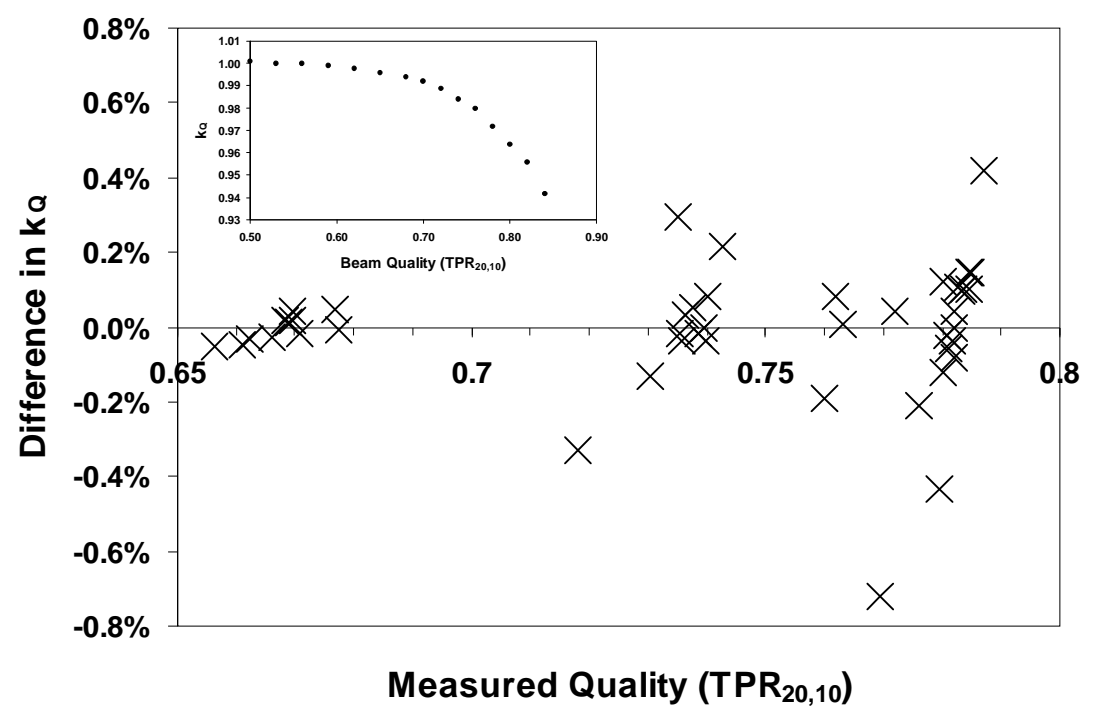

Figure 7. Percentage difference in $k_{Q}$ (derived from tabled values in TRS 398) between the value based on the local beam quality $\left(T P R_{20,10}\right)$ estimate, and that based on the measured quality. The inset shows the distribution of $k_{Q}$ for the CC13 chamber with $T P R_{20,10}$ provided in TRS 398.

The demographics for this study may not necessarily reflect the actual composition of treatment equipment in use in Australia and New Zealand. This study was combined with a Level III study of a pelvic phantom irradiation where accuracy and consistency of treatment was being investigated. There is thus significant potential for equipment bias. It could be expected that a centre would use their 'best' accelerator, the one they know to be reliable, the accelerator that had a lower clinical load or the one that was usually used for treating pelvic patients. It is also expected that accelerator output results could be biased due to centres verifying the calibration of their accelerators prior to the visit. The majority of centres in Australia and New Zealand maintain treatment equipment according to the recommendations of the $\mathrm{ACPSEM}^{28}$, which advises daily or weekly output assessment with a tolerance of $\pm 2 \%$. This is often in reference to a baseline measurement and not a complete absolute calibration so that this study should be identifying most systematic output variations. It is difficult to envisage any such study which would impose a surprise and random output assessment on a centre.

The distribution of output measurements do not suggest the influence of any systematic error (outside of measurement uncertainty) which could potentially be introduced from multiple sources - such as calibration of the reference chamber and intercomparison at $\mathrm{NMMH}$ and operator error (there was no significant difference in results between study members). It should also be noted that action limits warranting investigation (see Table 1) were never exceeded.

The distribution of beam qualities in Figure 2 reflects the tendency for the use of nominally $6 \mathrm{MV}, 10 \mathrm{MV}$ and $18 \mathrm{MV}$ beams in the associated in-phantom study. Analysis of the results of output measurements by T-Test indicates significant difference between the precision of reference dose measurements in each beam quality 'band' (i.e., for 6, 10 and $18 \mathrm{MV}$ beams; output standard deviations 0.006, 0.007 and 0.008 respectively - see Figure 4). The variation can possibly be attributed to an increase in sensitivity of output measurement to measurement and setup uncertainties with increasing energy. In Figure 7, this is demonstrated by evaluating the percentage difference between $k_{Q}$ derived from the local value of $\mathrm{TPR}_{20,10}$ and that measured during this study. Differences in $\mathrm{TPR}_{20,10}$ lead to increasingly larger differences in $k_{Q}$ with increasing energy, in addition to the inherent uncertainty in the TRS 398 calculated $k_{Q}$ values ${ }^{27}$. For the CC13 chamber, there is a rapid increase in change of correction factor $k_{Q}$ with increasing beam energy (see inset, Figure 7).

Figure 5 appears to show a decrease in the spread of output results with time during the study. Equipment types/manufacturers and beam qualities were uniformly represented throughout the study. As Figure 6 indicates, there was a steady progression of centres changing to the TRS 398 protocol during this study, though no correlation between output measurement and local calibration protocol could be identified. An increase in reference dose (and consequently accelerator output) is expected using TRS $398^{22}$ relative to the TRS $277^{23}$ air-kerma based protocol ${ }^{29}$. Although the output measured using TRS 398 was on average higher than the local value provided by centres that use TRS 277, this is well within the uncertainty in the measurement.

Additional data could have been collected during this study to assist identifying the cause of output errors or data trends, and it is recommended that these data are collected 


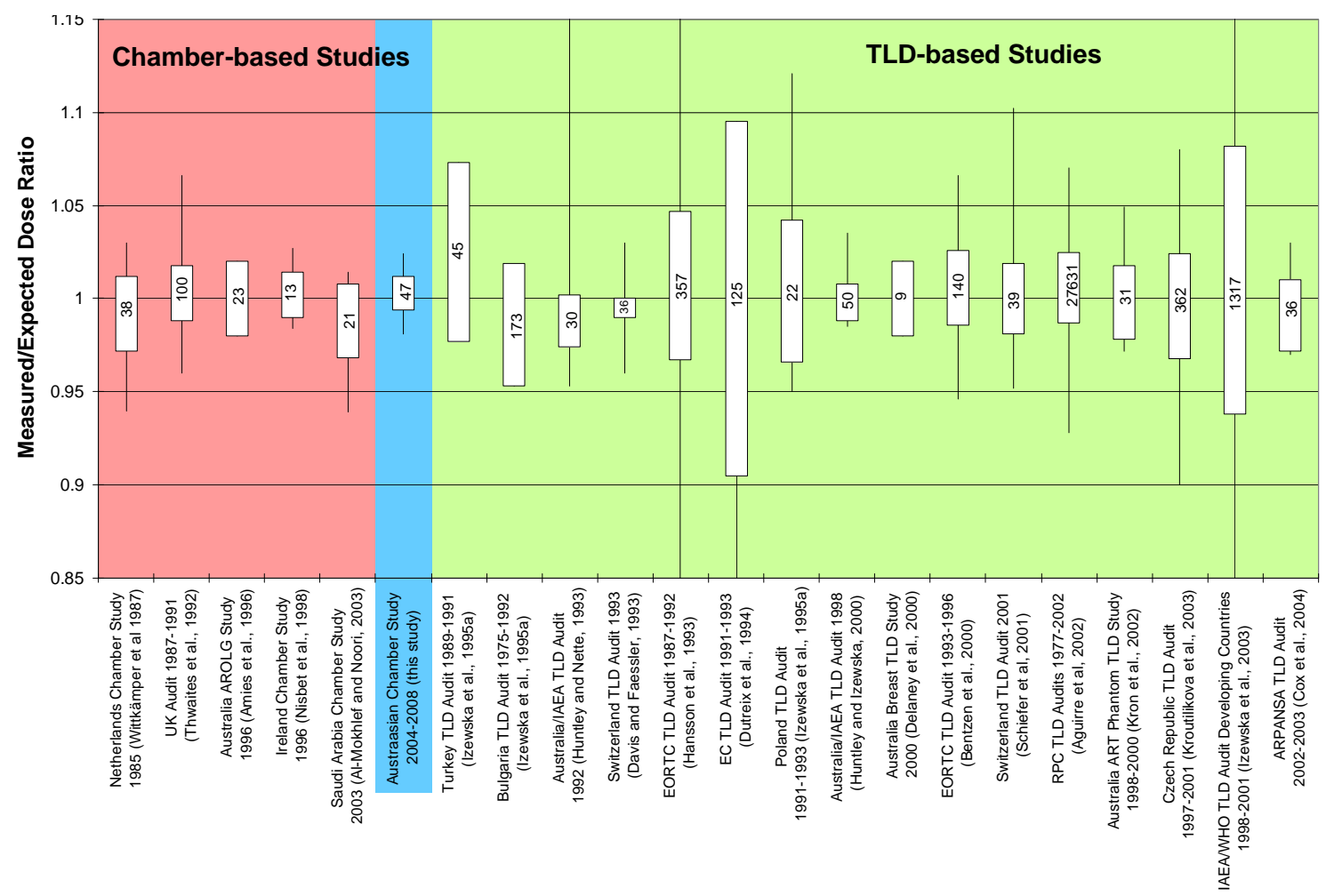

Figure 8. Comparison of Level I measurement results for photon studies published since 1985 (note - includes some ${ }^{60}$ Co beams as well as some repeat measurements on individual beams). Each data point has a box representing $\pm 1 S D$ about the mean and (where information was available) whiskers representing the minimum/maximum ratio ( ${ }^{O P} P_{\text {measured }} / O P_{\text {local }}$ up to maximum $\pm 15 \%$ ), and a number in each box which is the number of beams that measurements were made on in each case. References for each data point provided in horizontal-axis labels.

in future. These data include the age of the treatment unit, the details of equipment used by centres for their output calibrations, and an independent measurement of $P D D_{z_{\text {ref }}, z_{\text {norm }}}$ or $T P R_{z_{\text {ref }}, z_{\text {norm }}}$ to verify the reference depth relative dose. The latter was not possible with the equipment used as automated control of the chamber was not possible, and a manual measurement was not possible within the time typically allocated at a centre for these measurements.

The spread of values for $O P_{\text {local }}, O P_{\text {measured }}$ and $O P_{\text {measured }} / O P_{\text {local }}$ each has its own implications for dosimetric accuracy and dose delivery in the context of clinical trials in Australia and New Zealand. For $O P_{\text {local }}$, $80.4 \%$ of values were within $\pm 1 \%$ of unity and all values were within the $\pm 2 \%$ range recommended by the $\mathrm{ACPSEM}^{28}$, indicating good adherence to recommendations for accelerator outputs. The range of values for $O P_{\text {measured }}$ incorporates both the range in accelerator outputs at all centres as well as the uncertainty in the study measurements, including the consistency of application of the TRS 398 protocol by the study team. $67.4 \%$ of values were within $\pm 1 \%$ of unity, with only one value outside of $\pm 2 \%$. This indicates the potential spread of doses applied to patients during a study (ignoring higher-order variations of dose distributions in patients) who would otherwise be assumed to have received the specified prescription dose, placing a limit on the power of a trial to resolve the effects of any changes in applied dose.

$$
O P_{\text {measured }} / O P_{\text {local }} \text { incorporates actual accelerator output }
$$

with uncertainties and errors in output measurement both locally and by the study team. It is however, the value that is typically reported from intercomparison studies, with the term 'local' sometimes replaced with 'stated' or 'expected'. Figure 8 shows the results of this study in the context of other studies on clinical photon beams with results published since 1985 . This plot shows, for each study, the spread and range of measured beam outputs as a ratio to expected output. Note that these data include results for both ionization chamber and TLD based studies on accelerators as well as ${ }^{60} \mathrm{Co}$ units. Also shown is the number of beams measured in each case. It should be remembered that studies using chamber-based measurements have reduced measurement uncertainties and thus reduced standard deviations can be expected. The results for the current study provide a relatively narrow range of output measurements. 


\section{Conclusion}

An extensive accelerator output intercomparison has been carried out across Australian and New Zealand radiotherapy centres. The study has indicated consistency in accelerator output calibration, with results of this study comparing very favourably with previously published international intercomparisons.

The intercomparison method utilised an ionisation chamber with measurements performed by study members. The small-volume chamber used was ideal for transportation and parallel use in an anthropomorphic phantom, though it was important to fully assess the accuracy limitations of that chamber. TLD's have been used and are used extensively by many groups for intercomparisons via a mail-based exchange system. At the expense of accuracy, this is recommended for future Level I studies. However, in the context of a Level III study, a chamber measurement is the method of choice as it allows the reduction of uncertainties, the ability to exclude the contribution of accelerator output from subsequent dosimetric errors, and the ability to provide immediate feedback to the centre where the measurement was made.

\section{Glossary}

\begin{tabular}{|c|c|c|}
\hline ACPSEM & $\begin{array}{l}\text { Australasian College of Physical } \\
\text { Scientists and Engineers in Medicine }\end{array}$ & $T P R_{X, Y}$ \\
\hline ARPANSA & $\begin{array}{l}\text { Australian Radiation Protection and } \\
\text { Nuclear Safety Agency }\end{array}$ & $Z_{\text {norm }}$ \\
\hline EORTC & $\begin{array}{l}\text { European Organisation for Research } \\
\text { and Treatment of Cancer }\end{array}$ & \\
\hline ESTRO & $\begin{array}{l}\text { European Society for Therapeutic } \\
\text { Radiology and Oncology }\end{array}$ & $Z_{\text {ref }}$ \\
\hline IAEA & International Atomic Energy Agency & \\
\hline$k_{p o l}$ & Polarity effect correction factor & $Z_{\max }$ \\
\hline
\end{tabular}

Beam quality correction factor (at quality $Q$ relative to reference quality $\left.Q_{0}\right)$

$k_{s}$
$k_{T P}$
$N_{D, w}$

Ion recombination correction factor

Temperature and pressure correction factor

Chamber calibration coefficient at reference beam quality $Q_{0}$

NMMH The Newcastle Mater Misericordiae Hospital (now the 'Calvary Mater Newcastle') - the reference centre for this study

NRL The National Radiation Laboratory of New Zealand
$O P_{\text {local }}$

Value of accelerator output (Gy/nominal Gy) at each centre at $z_{\text {norm }}$ as supplied by local staff

$O P_{\text {measured }} \quad$ Value of accelerator output (Gy/nominal Gy) at each centre at $z_{\text {norm }}$ as measured by study staff

PSDL Primary Standards Dosimetry Laboratory

$P D D_{X . Y} \quad$ The percentage depth dose at depth $X$ relative to that at depth $Y$

Q General symbol to indicate beam quality. ' $Q_{0}$ ' indicates quality is for the reference beam, ${ }^{60} \mathrm{Co}$

RPC Radiological Physics Center, Houston, Texas

$S A D$

Source-to-axis distance. In terms of accelerator calibration, this refers to a configuration where $z_{r e f}$ is positioned at the accelerator isocentre

$S S D$

Source-to-surface distance. In terms of accelerator calibration, this refers to a configuration where the water surface remains fixed and $z_{r e f}$ is not at the accelerator isocentre

The tissue-phantom ratio at depth $X$ relative to that at depth $Y$

Depth (in millimetres) of the point on central axis where dose output is normalised (typically to 1 cGy per machine monitor unit)

Depth (in millimetres) of the point on central axis where the measurement of dose for establishing accelerator output is made (typically either $50 \mathrm{~mm}$ or $100 \mathrm{~mm}$ )

Depth (in millimetres) of the point on central axis of maximum dose

\section{Acknowledgements}

We are grateful to the Commonwealth of Australia Department of Health and Ageing, the New South Wales Cancer Institute, the Newcastle Mater Hospital and Sir Charles Gairdner Hospital for financial support. Many thanks to Oxford Scientific for providing equipment to undertake the measurements, to TROG for providing logistic support, and to the Department of Radiation Oncology including physicists at the NMMH for general tolerance, assistance and support. Thanks to Prof Tomas Kron for technical advice and to staff at ARPANSA and NRL for the provision of information and for their cooperation. Much appreciation is extended to staff at all participating centres. 


\section{References}

1. Kron, T., Hamilton, C., Roff, M. and Denham, J., Dosimetric intercomparison for two Australasian clinical trials using an anthropomorphic phantom, Int J Radiat Oncol Biol Phys, 52(2): 566-79, 2002.

2. Izewska, J., Georg, D., Bera, P., Thwaites, D., Arib, M., Saravi, M., Sergieva, K., Li, K., Yip, F.G., Mahant, A.K. and Bulski, W., A methodology for TLD postal dosimetry audit of high-energy radiotherapy photon beams in non-reference conditions, Radiother Oncol, 84(1): 67-74, 2007.

3. IAEA, Lessons learned from accidental exposures in radiotherapy, International Atomic Energy Agency, Safety Report Series N17, Vienna.

4. Bentzen, S.M., Bernier, J., Davis, J.B., Horiot, J.C., Garavaglia, G., Chavaudra, J., Johansson, K.A. and Bolla, M., Clinical impact of dosimetry quality assurance programmes assessed by radiobiological modelling of data from the thermoluminescent dosimetry study of the European Organization for Research and Treatment of Cancer, Eur J Cancer, 36(5): 615-20, 2000.

5. IAEA. IAEA TLD Postal Dose Audit Service. http://wwwnaweb.iaea.org/nahu/dmrp/tld.asp. 2007

6. Izewska, J., Andreo, P., Vatnitsky, S. and Shortt, K.R., The IAEA/WHO TLD postal dose quality audits for radiotherapy: a perspective of dosimetry practices at hospitals in developing countries, Radiother Oncol, 69(1): 91-7, 2003.

7. Izewska, J., Bera, P. and Vatnitsky, S., IAEA/WHO TLD postal dose audit service and high precision measurements for radiotherapy level dosimetry. International Atomic Energy Agency/World Health Organization, Rad Prot Dosim, 101(14): 387-92, 2002.

8. Izewska, J., Gajewski, R., Gwiazdowska, B., Kania, M. and Rostkowska, J., TLD postal dose intercomparison for megavoltage units in Poland, Radiother Oncol, 36(2): 143-52, 1995.

9. Svensson, H., Hanson, G.P. and Zsdanszky, K., The IAEA/WHO TL dosimetry service for radiotherapy centres 1969-87, Acta Oncologica, 29(4): 461-7, 1990.

10. Ferreira, I.H., Dutreix, A., Bridier, A., Chavaudra, J. and Svensson, H., The ESTRO-QUALity assurance network (EQUAL), Radiother Oncol, 55(3): 273-84, 2000.

11. Hansson, U., Johansson, K.A., Horiot, J.C. and Bernier, J., Mailed TL dosimetry programme for machine output check and clinical application in the EORTC radiotherapy group, Radiother Oncol, 29(2): 85-90, 1993.

12. Roue, A., Van Dam, J., Dutreix, A. and Svensson, H., [The EQUAL-ESTRO external quality control laboratory in France], Cancer Radiotherapie, 8 Suppl 1: S44-9, 2004.

13. Nisbet, A. and Thwaites, D.I., A dosimetric intercomparison of electron beams in UK radiotherapy centres, Phys Med Biol, 42(12): 2393-409, 1997.

14. Nisbet, A., Thwaites, D.I. and Sheridan, M.E., A dosimetric intercomparison of kilovoltage $X$-rays, megavoltage photons and electrons in the Republic of Ireland, Radiother Oncol, 48(1): 95-101, 1998.

15. Thwaites, D.I., Williams, J.R., Aird, E.G., Klevenhagen, S.C. and Williams, P.C., A dosimetric intercomparison of megavoltage photon beams in UK radiotherapy centres, Phys Med Biol, 37(2): 445-61, 1992.

16. Venables, K., The national trials QA centre. Who are we, what we do, and how we can help you, Scope, 15: 10-15, 2006.

17. Huntley, R. and Izewska, J., The 1998 Australian external beam radiotherapy survey and IAEA/WHO TLD postal dose quality audit, Aust Phys Eng Sci Med, 23(1): 21-9, 2000.
18. Amies, C., Rose, A., Metcalfe, P. and Barton, M., Multicentre dosimetry study of mantle treatment in Australia and New Zealand, Radiother Oncol, 40(2): 171-80, 1996.

19. Delaney, G., Beckham, W., Veness, M., Ahern, V., Back, M., Boyages, J., Fox, C., Graham, P., Jacob, G., Lonergan, D., Morgan, G., Pendlebury, S. and Yuile, P., Three-dimensional dose distribution of tangential breast irradiation: results of a multicentre phantom dosimetry study, Radiother Oncol, 57(1): 61-8, 2000.

20. Kron, T., Barnes, K. and O'Brien, P., Multicentre dosimetric comparison of photon-junctioning techniques in head and neck radiotherapy, Aust Radiol, 47(3): 289-94, 2003.

21. Cox, M., Huntley, R. and Webb, D., A TLD therapy dosimetry quality assurance program for Australia - Results 2002-2003, Aust Phys Eng Sci Med, 27(4): 264-265, 2004.

22. IAEA, Absorbed dose determination in external beam radiotherapy: An international code of practice for dosimetry based on standards of absorbed dose to water, International Atomic Energy Agency, Technical Report Series No. 398, Vienna.

23. IAEA, Absorbed dose determination in photon and electron beams: An international code of practice, International Atomic Energy Agency, Technical Report Series No. 277, Vienna.

24. Ebert, M.A., Harrison, K., Cornes, D., Howlett, S., Joseph, D.J., Kron, T., Hamilton, C.S. and Denham, J.W., A comprehensive Australasian multi-centre dosimetric intercomparison - issues, logistics and recommendations, Journal of Medical Imaging and Radiation Oncology, In press, 2008.

25. Ebert, M.A., Harrison, K., Denham, J.W., Cornes, D., Howlett, S. and Hamilton, C., QA of conformal radiotherapy for multi-centre radiotherapy trials in Australasia, in International Conference on Quality Assurance and New Technologies in Radiation Medicine. 2006, IAEA: Vienna, Austria.

26. Followill, D.S., Tailor, R.C., Tello, V.M. and Hanson, W.F., An empirical relationship for determining photon beam quality in TG-21 from a ratio of percentage depth doses, Medical Physics, 25: 1202-1205, 1998.

27. Castro, P., Garcia-Vicente, F., Minguez, C., Floriano, A., Sevillano, D., Pérez, L. and Torres, J.J., Study of the uncertainty in the determination of the absorbed dose to water during external beam radiotherapy calibration, Journal of Applied Clinical Medical Physics, 9(1): 70-86, 2008.

28. Millar, M., Cramb, J., Das, R., Ackerly, T., Brown, G., Webb, D., ACPSEM Position Paper Recommendations for the Safe Use of External Beams and Sealed Brachytherapy Sources in Radiation Oncology, Aust Phys Eng Sci Med, 20(3): 1-35, 1997.

29. Butler, D.J., Palmans, H. and Webb, D.V., Shift in absorbed dose for megavoltage photons when changing to TRS-398 in Australia, Aust Phys Eng Sci Med, 28(3): 159-164, 2005.

30. Wittkämper, F.W., Mijnheer, B.J. and van Kleffens, H.J., Dose intercomparison at the radiotherapy centres in The Netherlands. 1. Photon beams under reference conditions and for prostatic cancer treatment, Radiother Oncol, 9(1): 33-44, 1987.

31. Al-Mokhlef, J.M. and Noori, N., Quality assurance study for dosimetry of radiation therapy equipment in Saudi Arabia, Annals Of Saudi Medicine, 23(3-4): 148-151, 2003.

32. Huntley, R.B. and Nette, H.P., International Atomic Energy Agency/World Health Organisation TLD radiotherapy dosimetry intercomparison, Aust Phys Eng Sci Med, 16(1): 44-8, 1993. 
33. Davis, B. and Faessler, P., Quality audit of megavoltage radiotherapy units: intercomparison of dose at a reference point using a mailed TL-dosimetry system, Radiother Oncol, 28(1): 79-81, 1993.

34. Dutreix, A., Derreumaux, S., Chavaudra, J. and van der Schueren, E., Quality control of radiotherapy centres in Europe: beam calibration, Radiother Oncol, 32(3): 256-264, 1994.

35. Schiefer, H., Seelentag, W. and Stucki, G., Ein nationaler Dosimetrievergleich durch Postversand von TLDs. Swiss Society of Radiobiology and Medical Physics, 2001. http://www.sgsmp.ch/annrepA1.htm
36. Aguirre JF, T.R., Ibbott G, Stovall M, Hanson WF. Thermoluminscenence dosimetry as a tool for the remote verification of output for radiotherapy beams: 25 years of experience. in Proceedings of an International Symposium on Standards and Codes of Practice in Medical Radiation Dosimetry. Proceedings of an International Symposium on Standards and Codes of Practice in Medical Radiation Dosimetry. Vienna: IAEA, 2002.

37. Kroutilíková, D., Novotný, J. and Judas, L., Thermoluminescent dosimeters (TLD) quality assurance network in the Czech Republic, Radiother Oncol, 66(2): 235244, 2003. 